\title{
On Geometric Properties of the Decorrelator
}

\author{
Yonina C. Eldar
}

\begin{abstract}
In this letter we discuss geometric properties of the multiuser decorrelator receiver. In particular we show that the prevalent geometric interpretation of the correlating vectors comprising the decorrelator receiver in terms of orthogonal projections of the signature vectors is incorrect. The correct interpretation established in this letter is that the correlating vectors are oblique projections of the signature vectors onto appropriate spaces. Furthermore, we show that each branch of the decorrelator consists of an oblique projection onto the space spanned by the corresponding user's signature vector along the space spanned by the interferers, followed by a correlator with correlating vector equal to the corresponding user's signature vector.
\end{abstract}

Index Terms-Decorrelator, oblique projection, least-squares approximation, multiuser detection.

\section{INTRODUCTION}

I N A CDMA system each user transmits information by modulating its own signature vector. The received signal is modeled as the sum of the modulated signature vectors of the different users embedded in white additive Gaussian noise. Multiuser detectors for detection of CDMA signals try to mitigate the effect of the multiple-access interference (MAI) and the background noise. A simple linear receiver that only requires knowledge of the signature vectors is the decorrelator, introduced by Lupas and Verdu [1]. The aim of this letter is to provide some insight into the geometric properties of the decorrelator.

An important property of the decorrelator is that for linearly independent signature vectors, it totally eliminates the MAI. This is achieved (mathematically) by multiplying the received signal by the pseudo inverse of the signature matrix. We expect this important property to have a geometric interpretation as well. Attempts to describe the geometric properties of the decorrelator have been made by several authors (see, e.g., [2], [3]). The prevalent geometric interpretation is that each branch of the decorrelator consists of an orthogonal projection onto $\mathcal{V}$, which is the orthogonal complement of the space spanned by the interferers in the space spanned by the signatures, followed by a correlator with correlating vector equal to the desired user's signature vector.

It is obvious that by (orthogonally) projecting the received signal onto $\mathcal{V}$ the MAI is eliminated. However, if the desired user's signature vector does not lie in $\mathcal{V}$, then this orthogonal

Manuscript received April 22, 2001. The associate editor coordinating the review of this letter and approving it for publication was Dr. J. Ritcey. This work was supported in part through collaborative participation in the Advanced Sensors Collaborative Technology Alliance (CTA) sponsored by the U.S. Army Research Laboratory under Cooperative Agreement DAAD19-01-2-0008.

The author is with the Research Laboratory of Electronics, Massachusetts Institute of Technology, Cambridge, MA 02139 USA (e-mail: yonina@ @it.edu).

Publisher Item Identifier S 1089-7798(02)00972-9. projection will modify the component of the received signal due to the desired user. This is in contradiction to the well known fact that in the absence of noise and assuming unit power control the output of each branch of the decorrelator is equal to the symbol transmitted by the corresponding user.

In this letter we show that this widespread interpretation is incorrect. The correct interpretation is that each branch of the decorrelator consists of an oblique projection onto the space spanned by the desired user's signature vector along the space $\mathcal{V}^{\perp}$ spanned by the interferes, so that any component in $\mathcal{V}^{\perp}$ is nulled out while components in the former space are not modified, followed by a correlator with correlating vector equal to the desired user's signature vector. Since the oblique projection eliminates the interferers without modifying the component due to the desired user, in the absence of noise and assuming unit power control the output of each branch will indeed be equal to the symbol transmitted by the corresponding user.

\section{THE DECORRELATOR}

Consider an $M$-user white Gaussian synchronous CDMA system. The discrete-time model for the received signal $r$ is given by

$$
\mathbf{r}=\mathbf{S A b}+\mathbf{n}
$$

where $\mathbf{S}$ is the $N \times M$ matrix of columns $\mathbf{s}_{m}$ and $\mathbf{s}_{m} \in \mathbb{C}^{N}$ is the signature vector of the $m$ th user, $\mathbf{A}=\operatorname{diag}\left(A_{1}, \ldots, A_{M}\right)$ where $A_{m}>0$ is the received amplitude of the $m$ th user's signal, $\mathbf{b}$ is a vector of elements $b_{m}$ where $b_{m}$ is the symbol transmitted by the $m$ th user, and $\mathbf{n}$ is a white Gaussian zero mean noise vector with covariance $\mathbf{C}_{n}=\sigma^{2} \mathbf{I}$. We assume that all information sequences are equally likely, and that $\mathbf{s}_{m}^{*} \mathbf{s}_{m}=1$ for all $m$. For concreteness, we assume that $b_{m} \in\{1,-1\}$.

Based on the observed signal $\mathbf{r}$ a receiver is designed to demodulate the information transmitted by each user. The decorrelator receiver [1] is a linear multiuser receiver that exploits the structure of the MAI without knowledge of the channel parameters. It consists of multiplying the received signal by $\mathbf{S}^{\dagger}$, where $(\cdot)^{\dagger}$ denotes the Moore-Penrose pseudo inverse [4]. If the signature vectors $\mathbf{S}_{m}$ are linearly independent, then $\mathbf{S}^{\dagger}=$ $\left(\mathbf{S}^{*} \mathbf{S}\right)^{-1} \mathbf{S}^{*}$. The decorrelator receiver can be equivalently implemented using a correlation demodulator with correlating vectors $\mathbf{v}_{m}$ that are the columns of $\left(\mathbf{S}^{\dagger}\right)^{*}$, as depicted in Fig. 1. The $m$ th output of the decorrelator demodulator is then given by $a_{m}=\mathbf{v}_{m}^{*} \mathrm{r}$, and the $m$ th user's bit is detected as $\hat{b}_{m}=\operatorname{sgn}\left(a_{m}\right)$.

In this letter we present some geometric interpretations of the decorrelator. In particular, we show that the prevalent geometric interpretation of the vectors $\mathbf{v}_{m}$ in terms of orthogonal projections (see, e.g., [2]) is incorrect. 


\section{Minimum-Norm Least-SQuares ApPROXIMATION}

One way to derive the decorrelator is to seek an estimate $\hat{\mathbf{y}}$ of $\mathbf{y}=\mathbf{A b}$ [3], and then detect the $m$ th user's symbol based on $\hat{\mathbf{y}}$. Thus if e.g., $b_{m} \in\{-1,1\}$, then $\hat{b}_{m}=\operatorname{sgn}\left(y_{m}\right)$ where $y_{m}$ is the $m$ th component of $\hat{\mathbf{y}}$. In the absence of noise it follows from (1) that

$$
\mathbf{r}=\mathbf{S y} \text {. }
$$

If the signatures $\mathbf{s}_{m}$ are linearly independent, then the unique solution to (2) is $\mathbf{y}=\left(\mathbf{S}^{*} \mathbf{S}\right)^{-1} \mathbf{S}^{*} \mathbf{r}=\mathbf{S}^{\dagger} \mathbf{r}$. If, on the other hand, the vectors $\mathbf{S}_{m}$ are linearly dependent, then there are infinitely many solutions to (2).

When there is noise present, (2) becomes the approximate relation $\mathrm{r} \approx \mathrm{Sy}$. A reasonable approach then is to seek a vector $\hat{\mathbf{y}}$ that renders $\mathbf{S} \hat{\mathbf{y}}$ as close as possible to $\mathbf{r}$. Thus, we choose $\hat{\mathbf{y}}$ to minimize the least-squares error

$$
\varepsilon_{\mathrm{LS}}(\mathbf{y})=(\mathbf{r}-\mathbf{S y})^{*}(\mathbf{r}-\mathbf{S y}) .
$$

In [3, p. 241] it is claimed that the minimizing $\hat{\mathbf{y}}$ is

$$
\hat{\mathbf{y}}=\mathbf{S}^{\dagger} \mathbf{r} \text {. }
$$

It is important to note that when the vectors $\mathbf{s}_{m}$ are linearly dependent, $\hat{\mathbf{y}}$ given by (4) is not the only vector that minimizes (3). Indeed, let

$$
\tilde{\mathbf{y}}=\hat{\mathbf{y}}+\mathbf{y}^{\prime}
$$

where $\mathbf{y}^{\prime}$ is any vector in the null space $\mathcal{N}(\mathbf{S})$ of $\mathbf{S}$. Then since $\mathbf{S y}^{\prime}=0, \mathbf{S} \tilde{\mathbf{y}}=\mathbf{S} \hat{\mathbf{y}}$ and $\varepsilon_{\mathrm{LS}}(\tilde{\mathbf{y}})=\varepsilon_{\mathrm{LS}}(\hat{\mathbf{y}})$, so that $\tilde{\mathbf{y}}$ also minimizes (3). However, $\hat{\mathbf{y}}$ given by (4) has the property that it has the minimal possible norm from all vectors $\tilde{\mathbf{y}}$ that minimize the least-squares error. This result follows from the fact that any $\tilde{\mathbf{y}}$ that minimizes (3) has the form given by (5). Furthermore, from (4) it follows that $\hat{\mathbf{y}}$ is in the range space of $\mathbf{S}^{\dagger}$ which is equal to $\mathcal{N}(\mathbf{S})^{\perp}$. Thus, $\hat{\mathbf{y}}^{*} \mathbf{y}^{\prime}=0$ for any $\mathbf{y}^{\prime} \in \mathcal{N}(\mathbf{S})$ and $\|\tilde{\mathbf{y}}\|^{2}=\|\hat{\mathbf{y}}\|^{2}+\left\|\mathbf{y}^{\prime}\right\|^{2} \geq\|\hat{\mathbf{y}}\|^{2}$, with equality if and only if $\mathbf{y}^{\prime}=0$ so that $\tilde{\mathbf{y}}=\hat{\mathbf{y}}$.

We therefore conclude that the output of the decorrelator demodulator $\mathbf{S}^{\dagger} \mathbf{r}$ is the minimum norm least-squares approximation to $\mathbf{y}=\mathbf{A b}$.

\section{Correlation Demodulator REPRESENTATION}

We now provide a geometric interpretation of the correlating vectors $\mathbf{v}_{m}$ of Fig. 1. For simplicity of exposition we assume throughout this section that the signature vectors $\mathbf{s}_{m}$ are linearly independent.

In [2] it is claimed that $\mathbf{v}_{1}$ is the orthogonal projection of $\mathbf{s}_{1}$ onto the orthogonal complement of the space spanned by the vectors $\left\{\mathbf{s}_{m}, 2 \leq m \leq M\right\}$ in the space spanned by the signature vectors, denoted $\mathcal{V}$. In [3] it is claimed that $\mathbf{v}_{1}$ is the projection of $\mathbf{s}_{1}$ onto $\mathcal{V}$, however the type of projection is not specified. In this section we show that the correct result is that $\mathbf{v}_{1}$ is the oblique projection of $\mathbf{s}_{1}$ onto $\mathcal{V}$ along $\mathcal{W}$, where $\mathcal{W}$ is the orthogonal complement of the space spanned by the vector $\mathbf{s}_{1}$.

We now demonstrate that $\mathbf{v}_{1}$ cannot be equal to $P_{\mathcal{V}} \mathbf{s}_{1}$, where $P \mathcal{V}$ is the orthogonal projection onto $\mathcal{V}$, using two different

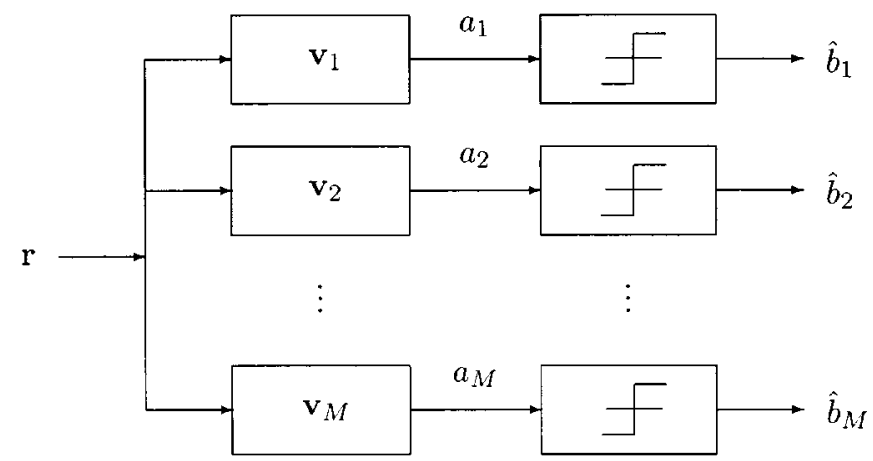

Fig. 1. Decorrelator receiver for the case $b_{m} \in\{1,-1\}$.

methods. First we note that if $\mathbf{v}_{1}=P_{\mathcal{V}} \mathbf{s}_{1}$, then we must have that $\left\|\mathbf{v}_{1}\right\|^{2} \leq\left\|\mathbf{s}_{1}\right\|^{2}$. But

$$
\left\|\mathbf{v}_{1}\right\|^{2}=\left[\left(\mathbf{S}^{*} \mathbf{S}\right)^{-1}\right]_{1} \mathbf{S}^{*} \mathbf{S}\left[\left(\mathbf{S}^{*} \mathbf{S}\right)^{-1}\right]_{1}=\left[\left(\mathbf{S}^{*} \mathbf{S}\right)^{-1}\right]_{11}
$$

where $[\cdot]_{m k}$ and $[\cdot]_{m}$ denote the $m k$ th element and the $m$ th column of a matrix, respectively. From (6) it follows that $\left\|\mathbf{v}_{1}\right\|^{2}$ is not necessarily smaller than $\left\|\mathbf{s}_{1}\right\|^{2}$. For example, consider a two user system with

$$
\mathbf{S}^{*} \mathbf{S}=\left[\begin{array}{cc}
1 & a \\
a & 1
\end{array}\right]
$$

where $0<a<1$. Then $\left\|\mathbf{v}_{1}\right\|^{2}=\left[\left(\mathbf{S}^{*} \mathbf{S}\right)^{-1}\right]_{11}=1 /\left(1-a^{2}\right)>$ $1=\left\|\mathbf{s}_{1}\right\|^{2}$, so that $\mathbf{v}_{1}$ cannot be equal to $P \mathcal{V} \mathbf{s}_{1}$.

Next, recall that in the absence of noise $\mathbf{r}=\mathbf{S A b}$ and $\mathbf{S}^{\dagger} \mathbf{r}=$ Ab so that $a_{1}=\mathbf{v}_{1}^{*} \mathbf{r}=A_{1} b_{1}$. If $\mathbf{v}_{1}=P_{\mathcal{L}} \mathbf{s}_{1}$, then

$$
\mathbf{v}_{1}^{*} \mathbf{r}=\mathbf{s}_{1}^{*} P \mathcal{\nu} .
$$

Now

$$
P_{\mathcal{V}} \mathrm{r}=P_{\mathcal{V}} \mathbf{S A b}=A_{1} b_{1} P_{\mathcal{V}} \mathbf{s}_{1}
$$

since $P_{\mathcal{\nu}} \mathbf{s}_{m}=0,2 \leq m \leq M$. Substituting (8) into (9) we conclude that

$$
\mathbf{v}_{1}^{*} \mathbf{r}=A_{1} b_{1} \mathbf{s}_{1}^{*} P_{\mathcal{V}} \mathbf{s}_{1}
$$

which is equal to $a_{1}=A_{1} b_{1}$ only in the case that $\mathbf{s}_{1}$ is orthogonal to all the other signature vectors. Thus, in general $\mathbf{v}_{1} \neq$ $P \mathcal{L} \mathrm{s}_{1}$.

To derive the correct geometric interpretation we first show that $\mathbf{v}_{1} \in \mathcal{V}$. Indeed

$$
\mathbf{S}^{*} \mathbf{v}_{1}=\mathbf{S}^{*} \mathbf{S}\left[\left(\mathbf{S}^{*} \mathbf{S}\right)^{-1}\right]_{1}=[\mathbf{I}]_{1}
$$

so that $\mathbf{v}_{1}$ is orthogonal to $\mathbf{s}_{m}, 2 \leq m \leq M$, and consequently $\mathbf{v}_{1} \in \mathcal{V}$. Since $\mathcal{V}$ is a one-dimensional space it is spanned by any nonzero vector in $\mathcal{V}$. In particular, let $\tilde{\mathbf{v}}=P_{\mathcal{V}} \mathbf{s}_{1}$. Since the vectors $\mathbf{s}_{m}$ are linearly independent, $\tilde{\mathbf{v}} \neq 0$ and $\tilde{\mathbf{v}}$ spans $\mathcal{V}$. Now, since $\mathbf{v}_{1} \in \mathcal{V}$ we have that $\mathbf{v}_{1}=\alpha \tilde{\mathbf{v}}=\alpha P_{\mathcal{V}} \mathbf{s}_{1}$ for some $\alpha$. To determine $\alpha$ we note that (11) implies that $\mathbf{s}_{1}^{*} \mathbf{v}_{1}=\alpha \mathbf{s}_{1}^{*} P \nu \mathbf{s}_{1}=$ 1 , so that $\alpha=1 / \mathbf{s}_{1}^{*} P_{\mathcal{V}} \mathbf{s}_{1}$. Thus,

$$
\mathbf{v}_{1}=\frac{1}{\mathbf{s}_{1}^{*} P_{\mathcal{V}} \mathbf{s}_{1}} P_{\mathcal{V}} \mathbf{s}_{1}
$$




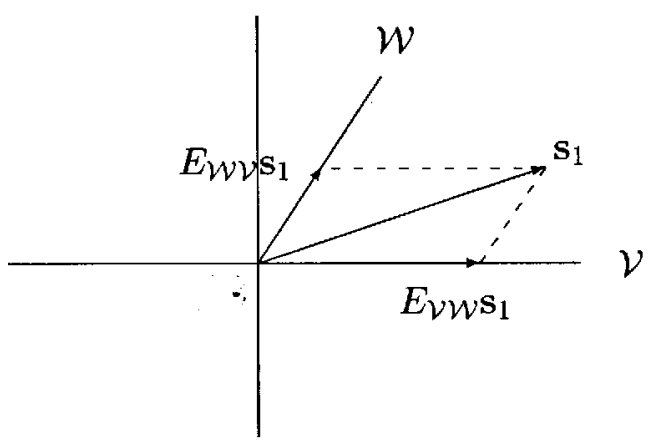

Fig. 2. Decomposition of $\mathbf{s}_{1}$ into its components in $\mathcal{V}$ and in $\mathcal{W}$ given by $E_{\mathcal{W}} \mathbf{s}_{1}$ and $E_{\mathcal{W} \mathcal{V}} \mathbf{s}_{1}$, respectively.

which in general is not equal to $P \mathcal{V} \mathbf{s}_{1}$, except in the special case that $\mathbf{s}_{1}$ is orthogonal to $\mathbf{s}_{m}, 2 \leq m \leq M$, in which case $P_{2} \mathbf{s}_{1}=$ $\mathbf{s}_{1}$.

We now show that (12) implies that $\mathbf{v}_{1}$ is the oblique projection of $\mathbf{s}_{1}$ onto $\mathcal{V}$ along $\mathcal{W}$, denoted by ${ }^{1} E_{\mathcal{W}}$ [5]. To this end we first show that

$$
E_{\mathcal{V} \mathcal{W}}=\frac{1}{\mathbf{s}_{1}^{*} P_{\mathcal{V}} \mathbf{s}_{1}} P_{\mathcal{V}} \mathbf{s}_{1} \mathbf{s}_{1}^{*}
$$

From the definition of an oblique projection, $E_{\mathcal{V}} \mathcal{W}$ is the unique operator satisfying

$$
\begin{array}{ll}
E_{\mathcal{V}} \mathbf{v}=\mathbf{v}, & \text { for any } \mathbf{v} \in \mathcal{V} \\
E_{\mathcal{V}} \mathbf{w}=0, & \text { for any } \mathbf{w} \in \mathcal{W} .
\end{array}
$$

We now show that (13) satisfies these conditions. Let $\mathbf{v}$ be an arbitrary vector in $\mathcal{V}$. Then $\mathbf{v}=a \tilde{\mathbf{v}}=a P_{\mathcal{V}} \mathbf{s}_{1}$ for some constant $a$, and

$$
E_{\mathcal{V} W} \mathbf{v}=\frac{a}{\mathbf{s}_{1}^{*} P_{\mathcal{V}} \mathbf{s}_{1}} P_{\mathcal{V}} \mathbf{s}_{1} \mathbf{s}_{1}^{*} P_{\mathcal{V}} \mathbf{s}_{1}=a P_{\mathcal{V}} \mathbf{s}_{1}=\mathbf{v}
$$

Now, let $\mathbf{w}$ be an arbitrary vector in $\mathcal{W}$. Then

$$
E_{\mathcal{V} \mathcal{W}} \mathbf{w}=\frac{a}{\mathbf{s}_{1}^{*} P_{\mathcal{V}} \mathbf{s}_{1}} P_{\mathcal{V}} \mathbf{s}_{1} \mathbf{s}_{1}^{*} \mathbf{w}=0
$$

since $\mathbf{w}$ is orthogonal to $\mathbf{s}_{1}$. Combining (15) and (16) we conclude that $E_{\mathcal{V}}$ is given by (13). We then have

$$
E_{\mathcal{V}} \mathbf{s}_{1}=\frac{1}{\mathbf{s}_{1}^{*} P_{\mathcal{V}} \mathbf{s}_{1}} P \mathcal{V} \mathbf{s}_{1} \mathbf{s}_{1}^{*} \mathbf{s}_{1}=\frac{1}{\mathbf{s}_{1}^{*} P_{\mathcal{V}} \mathbf{s}_{1}} P_{\mathcal{V}} \mathbf{s}_{1}=\mathbf{v}_{1} \text {. }
$$

Thus, $\mathbf{v}_{1}$ is the oblique projection of $\mathbf{s}_{1}$ onto $\mathcal{V}$ along $\mathcal{W}$. Fig. 2 depicts the decomposition of $\mathbf{s}_{1}$ into its component in $\mathcal{V}, \mathbf{v}_{1}=$ $E_{\mathcal{W}} \mathrm{s}_{1}$, and its component in $\mathcal{W}, E_{\mathcal{W}} \mathbf{s}_{1}$.

\footnotetext{
${ }^{1}$ An oblique projection is a projection operator $E$ satisfying $E^{2}=E$ that is not necessarily Hermitian. The notation $E_{\mathcal{Y} \mathcal{W}}$ denotes an oblique projection with range space $\mathcal{V}$ and null space $\mathcal{W}$. If $\mathcal{V}=\mathcal{W} \perp$, then $E_{\mathcal{V} \mathcal{W}}$ is an orthogonal projection onto $\mathcal{V}$.
}

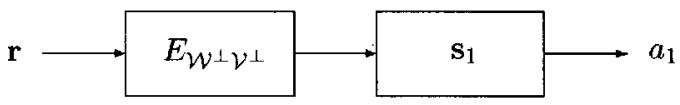

Fig. 3. Equivalent representation of the first branch of the decorrelator demodulator.

Using (17) we can express the output of the first branch of the decorrelator demodulator as $a_{1}=\mathbf{v}_{1}^{*} \mathbf{r}=\mathbf{s}_{1}^{*} E_{\mathcal{V}}^{*} \mathbf{r}$. From the properties of the oblique projection $E_{\mathcal{V}}^{*} \mathcal{Y}=E_{\mathcal{W} \perp \mathcal{V} \perp}$ which is the oblique projection onto $\mathcal{W}^{\perp}$ along $\mathcal{V}^{\perp}$, where $\mathcal{W}^{\perp}$ is the space spanned by $\mathbf{s}_{1}$ and $\mathcal{V}^{\perp}$ is the space spanned by the vectors $\left\{\mathbf{s}_{m}, 2 \leq m \leq M\right\}$. Thus the output $a_{1}$ of the decorrelator is obtained by first projecting $\mathbf{r}$ onto $\mathcal{W}^{\perp}$ along $\mathcal{V}^{\perp}$, and then correlating the result with $\mathbf{s}_{1}$, as depicted in Fig. 3. This result is intuitively pleasing. The oblique projection $E_{\mathcal{W} \perp \mathcal{V} \perp \text { nulls out }}$ any component in $\mathcal{V}^{\perp}$ which is the space spanned by the interferers, while not effecting any component in $\mathcal{W}^{\perp}$ which is the space spanned by $\mathbf{s}_{1}$. Thus $E_{\mathcal{W} \perp \mathcal{V} \perp \mathbf{s}_{m}}=\delta_{1 m} \mathbf{s}_{m}, 1 \leq m \leq M$. Note, that while $P_{\mathcal{V}} \mathbf{s}_{m}=0,2 \leq m \leq M, P_{\mathcal{V}} \mathbf{s}_{1} \neq \mathbf{s}_{1}$ in general. Now, in the absence of noise we have

$$
\mathbf{v}_{1}^{*} \mathbf{r}=\mathbf{s}_{1}^{*} E_{\mathcal{W} \perp \mathcal{V} \perp} \mathbf{r}=\mathbf{s}_{1}^{*} E_{\mathcal{W} \perp \mathcal{V} \perp} \mathbf{S A b}=A_{1} b_{1}
$$

as we expect.

Therefore, the correct interpretation of the decorrelator is that it projects the received signal onto the space spanned by the desired user's signature vector along the direction spanned by the interferers, and then correlates the projection with the desired user's signature vector.

\section{CONCLUSION}

We provided some geometrical interpretations of the decorrelator receiver. We first showed that the vector output of the decorrelator demodulator is the minimum norm least-squares approximation to $\mathbf{A b}$. We then established that each branch of the decorrelator can be implemented by projecting the received signal onto the space spanned by the signature vector of the corresponding user, along the space spanned by the interferers' signature vectors. The projected received signal is then correlated with the corresponding user's signature vector.

\section{REFERENCES}

[1] R. Lupas and S. Verdu, "Linear multiuser detectors for synchronous code-division multiple-access channels," IEEE Trans. Inform. Theory, vol. 35, pp. 123-136, Jan. 1989.

[2] D. N. C. Tse and S. V. Hanly, "Linear multiuser receivers: Effective interference, effective bandwidth and user capacity," IEEE Trans. Inform. Theory, vol. 45, pp. 641-657, Mar. 1999.

[3] S. Verdu, Multiuser Detection. New York: Cambridge Univ. Press, 1998.

[4] G. H. Golub and C. F. Van Loan, Matrix Computations, 3rd ed. Baltimore, MD: Johns Hopkins Univ. Press, 1996.

[5] S. Kayalar and H. L. Weinert, "Oblique projections: Formulas, algorithms, and error bounds," Math. Contr. Signals Syst., vol. 2, no. 1, pp. $33-45,1989$. 